OPEN ACCESS

Edited by: Giuseppantonio Maisetta, University of Pisa, Italy

Reviewed by:

Jianfeng Wang,

Jilin University, China

Rohitashw Kumar

University at Buffalo, United States

*Correspondence.

Austin S. Nuxoll

nuxollas@unk.edu

Specialty section:

This article was submitted to Microbial Physiology and Metabolism,

a section of the journal

Frontiers in Microbiology

Received: 28 August 2019 Accepted: 19 November 2019 Published: 05 December 2019

Citation

Nabb DL, Song S, Kluthe KE, Daubert TA, Luedtke BE and Nuxoll AS (2019) Polymicrobial

Interactions Induce Multidrug Tolerance in Staphylococcus aureus

Through Energy Depletion.

Front. Microbiol. 10:2803.

doi: 10.3389/fmicb.2019.02803

\section{Polymicrobial Interactions Induce Multidrug Tolerance in Staphylococcus aureus Through Energy Depletion}

Dan L. Nabb, Seoyoung Song, Kennedy E. Kluthe, Trevor A. Daubert, Brandon E. Luedtke and Austin S. Nuxoll*

Department of Biology, University of Nebraska at Kearney, Kearney, NE, United States

Staphylococcus aureus is responsible for a high number of relapsing infections, which are often mediated by the protective nature of biofilms. Polymicrobial biofilms appear to be more tolerant to antibiotic treatment, however, the underlying mechanisms for this remain unclear. Polymicrobial biofilm and planktonic cultures formed by S. aureus and Candida albicans are 10- to 100-fold more tolerant to oxacillin, vancomycin, ciprofloxacin, delafloxacin, and rifampicin compared to monocultures of $S$. aureus. The possibility of $C$. albicans matrix components physically blocking antibiotic molecules from reaching $S$. aureus was ruled out as oxacillin, ciprofloxacin, delafloxacin, and rifampicin were able to diffuse through polymicrobial biofilms. Based on previous findings that $S$. aureus forms drug tolerant persister cells through ATP depletion, we examined nutrient deprivation by determining glucose availability, which indirectly correlates to ATP production via the tricarboxylic acid (TCA) cycle. Using an extracellular glucose assay, we confirmed that S. aureus and C. albicans polymicrobial cultures depleted available glucose faster than the respective monocultures. Supporting this finding, S. aureus exhibited decreased TCA cycle activity, specifically fumarase expression, when grown in the presence of $C$. albicans. In addition, $S$. aureus grown in polymicrobial cultures displayed 2.2-fold more cells with low membrane potential and a $13 \%$ reduction in intracellular ATP concentrations than in monocultures. Collectively, these data demonstrate that decreased metabolic activity through nutrient deprivation is a mechanism for increased antibiotic tolerance within polymicrobial cultures.

Keywords: Staphylocccus aureus, persister, Candida albicans, energy depletion, polymicrobial

\section{INTRODUCTION}

Globally, 1 in 20 patients are currently suffering from a nosocomial infection (Zarb et al., 2012; Koch et al., 2015a,b), with Staphylococcus aureus being a prevalent organism associated with these infections (Hassoun et al., 2017). S. aureus is a leading cause of infective endocarditis, osteomyelitis, skin and soft tissue infections, and prosthetic device-related infections (Tong et al., 2015). 
A number of $S$. aureus mediated infections can be attributed to the contamination of the device surface with a biofilm (Wolcott et al., 2010). Interestingly, biofilm mediated S. aureus infections are difficult to eradicate, yet are caused primarily by drug-susceptible strains (Conlon, 2014; Ericson et al., 2015). Moreover, in polymicrobial biofilms, S. aureus is interacting with other pathogens, including the fungus Candida albicans. Polymicrobial infections are of concern as they result in a higher mortality rate than monomicrobial infections (Goetghebeur et al., 2007; Perlroth et al., 2007). However, underlying mechanisms for these observations remain inconclusive (McKenzie, 2006; Lin et al., 2010; Fengcai et al., 2015; Royo-Cebrecos et al., 2017). Polymicrobial biofilms have been reported to increase pathogen virulence, antibiotic resistance, and biofilm robustness (Harriott and Noverr, 2009, 2010, 2011; Kong et al., 2016). More specifically, tolerance to vancomycin in polymicrobial biofilms with C. albicans through an increase in biofilm robustness due to the extracellular matrix products secreted by the C. albicans, which restricted vancomycin penetration into the biofilm (Singh et al., 2010; Kong et al., 2016). However, similar results were found in $S$. aureus monomicrobial biofilms treated with vancomycin (Singh et al., 2010); therefore, it is difficult to make any direct inferences about the underlying causes of tolerance to antibiotics.

Until recently, literature on the mechanisms of persister cell formation was limited to two themes, toxin-antitoxin (TA) modules and stringent response (Lewis, 2010; Maisonneuve et al., 2013). However, it was recently demonstrated that TA modules did not have a role in $S$. aureus persister cell formation (Conlon et al., 2016), and the stringent response, when disrupted in $S$. aureus had no effect on persister formation. Instead, it was observed that $S$. aureus cells exhibiting lower intracellular ATP had increased persister formation and tolerance to antibiotics (Conlon et al., 2016). Additional work confirmed an association between decreased metabolic activity in the TCA cycle and membrane potential with $S$. aureus persister formation (Wang et al., 2018). The metabolic status of $S$. aureus and nutrient acquisition has become of interest for explaining bacterial survival during chronic infection and more recently has been associated with antibiotic tolerance in S. aureus. Nutrients such as amino acids, iron, nitrogen, and carbon metabolism have been a focal point of recent in vivo investigations (Haley and Skaar, 2012; Halsey et al., 2016; Spahich et al., 2016). While glucose is required for initial infection, in mature abscesses, non-preferred carbon sources are often a limiting factor (Kelly and O'Neill, 2015; Spahich et al., 2016; Thurlow et al., 2018). Similarly, bacteria appear to form more robust biofilms when grown in the presence of abundant glucose. As the biofilm matures, glucose is exhausted leading to the formation of persisters (Amato and Brynildsen, 2014). These environments provide examples where glucose is required for initial establishment of infection, but as the infection progresses glucose availability becomes less important. Furthermore, nutrient sparse environments are frequently associated with relapsing chronic infection following antibiotic therapy. This points to a need for further exploration of the role of nutrient depletion in relapsing infections.

In this study, glucose exhaustion and the subsequent decrease in energy availability was explored as a mechanism for multidrug tolerance within $S$. aureus and $C$. albicans polymicrobial cultures. It was found that polymicrobial cultures depleted glucose more rapidly compared to monomicrobial cultures. Additionally, S. aureus grown in polymicrobial cultures demonstrated decreased intracellular ATP concentrations as well as lower membrane potential when compared to cultures lacking C. albicans. Evidence for increased antibiotic tolerance within polymicrobial cultures due to matrix composition or biomolecules secreted by $C$. albicans was not found. Overall, these studies highlight the importance of metabolism in bacterial persistence, and demonstrate a potential mechanism for relapse in polymicrobial infection following antibiotic treatment.

\section{MATERIALS AND METHODS}

\section{Strains and Growth Conditions}

The methicillin susceptible $S$. aureus strain HG003 was used in all assays (Herbert et al., 2010). The community acquired C. albicans strain SC5314 was used for all experiments (Gillum et al., 1984; Odds et al., 2004). For experiments demonstrating this phenotype occurs across staphylococcal species, Staphylococcus epidermidis 1457, S. aureus UAMS1 , and $S$. aureus JE2 were used. S. epidermidis was grown to late $\log \left(\sim 1 \times 10^{9} \mathrm{CFU} / \mathrm{mL}\right)$ as this species is more sensitive to antibiotics an eradication occurs at early log phase. S. aureus JE2 is highly tolerant to antibiotics in later phases of growth and therefore assays were performed in early $\log \left(3 \times 10^{7} \mathrm{CFU} / \mathrm{mL}\right)$. S. aureus UAMS-1 and HG003 are similar in persister formation and assays were performed in mid-log $\left(2-5 \times 10^{8} \mathrm{CFU} / \mathrm{mL}\right)$. SC5314 was grown to $\sim 3 \times 10^{6} \mathrm{CFU} / \mathrm{mL}$ for each biofilm and time-dependent kill assay where polymicrobial cultures were utilized. The Pspa:gfp plasmid was provided by Kim Lewis (Conlon et al., 2016). For construction of the PfumC:gfp reporter, the promoter of fumC was amplified (5'-gggccogaattcttgatgatgttaatgcgcaaa- $3^{\prime}$ and $5^{\prime}$ gggccctctagatcaatttctcccttatcac- $3^{\prime}$ ) and cloned upstream of $g f p$ into the EcoRI and XbaI sites in pALC1434 (Cheung et al., 1998). Once cloned, PfumC:gfp was electroporated into S. aureus RN4220 and subsequently transduced into HG003 using $\Phi 11$ phage. Unless otherwise stated, all growth steps and timedependent kill assays were grown in $3 \mathrm{~mL}$ Tryptic Soy Broth (TSB) at $37^{\circ} \mathrm{C}$ at $225 \mathrm{rpm}$ in $14 \mathrm{~mL}$ snap cap tubes.

\section{6-Well Static Biofilm Tolerance Assays}

Overnight cultures of $S$. aureus were diluted 1:1000 and C. albicans overnight cultures were diluted $1: 100$ in $100 \mu \mathrm{L}$ of TSB within a 96-well polystyrene flat-bottom plate. Plates were incubated statically for $8 \mathrm{~h}$. Non-adherent cells were washed with $1 \% \mathrm{NaCl}$, fresh TSB was added, and biofilms were subsequently challenged with antibiotics (10-100 $\times$ MIC) for $24 \mathrm{~h}$. MICs were previously determined for HG003: ciprofloxacin $(0.5 \mu \mathrm{g} / \mathrm{mL})$, 
gentamicin (1 $\mu \mathrm{g} / \mathrm{mL})$, oxacillin $(0.5 \mu \mathrm{g} / \mathrm{mL})$, vancomycin (1 $\mu \mathrm{g} / \mathrm{mL})$, rifampicin $(0.008 \mu \mathrm{g} / \mathrm{mL})$. Finally, biofilms were solubilized and plated on TSA containing amphotericin B (25 $\mu \mathrm{g} / \mathrm{mL})$ using a standard serial dilution technique. Error bars represent the standard deviation and statistical significance was determined using a $t$-test, $P \leq 0.05$.

\section{Planktonic Time-Dependent Kill Assays}

Planktonic cultures were grown to mid-exponential phase in $3 \mathrm{~mL}$ TSB and challenged with antibiotics (10-100× MIC) as described previously (Conlon et al., 2016; Zalis et al., 2019). Cultures were placed in a shaking incubator at $225 \mathrm{rpm}$ at $37^{\circ} \mathrm{C}$. $100 \mu \mathrm{L}$ aliquots were removed from samples, washed to remove antibiotic, and surviving bacteria were enumerated at 18, 24, 48 , and $72 \mathrm{~h}$ by serial dilution and plating on TSA containing amphotericin B $(25 \mu \mathrm{g} / \mathrm{mL})$.

\section{Antibiotic Diffusion Through Mono- and Polymicrobial Biofilms}

Polycarbonate filters $(13 \mathrm{~mm})$ were sterilized by UV light for 30 min per side and placed on a TSA plate. Overnight S. aureus cultures were diluted $1: 1000, C$. albicans overnight cultures were diluted 1:100 in TSB. $100 \mu \mathrm{L}$ of this solution was placed onto the filter and grown statically for $24 \mathrm{~h}$. Biofilms were placed on fresh TSA plates seeded with $1 \times 10^{6} \mathrm{CFU}$ S. aureus. A $13 \mathrm{~mm}$ polycarbonate disk was placed on the biofilm, followed by a diffusion disk. Each respective antibiotic ( $1 \mathrm{mg} / \mathrm{mL}$ ciprofloxacin, $10 \mathrm{mg} / \mathrm{mL}$ oxacillin, $1 \mathrm{mg} / \mathrm{mL}$ rifampicin, $10 \mathrm{mg} / \mathrm{mL}$ vancomycin) was added $(10 \mu \mathrm{L})$ to the disk and plates were incubated for $24 \mathrm{~h}$. The diameter of the zone of inhibition was then measured in millimeters. The average and standard deviation was obtained from biological triplicates. Significance was determined using a $t$-test, $P \leq 0.05$.

\section{Visualization of Antibiotic Diffusion Throughout a Biofilm Using Confocal Scanning Laser Microscopy}

To visualize antibiotic diffusion through single and polymicrobial biofilms, fluorescently labeled vancomycin and delafloxacin were used as described previously with modification (Pereira et al., 2007). Biofilms were grown on 8 -chambered glass coverslips (cat. 154941, MatTek Co.) for $24 \mathrm{~h}$ at $37^{\circ} \mathrm{C}$ statically in TSB containing $1 \%$ glucose. Following incubation, non-adherent cells were washed gently with $1 \% \mathrm{NaCl}$, and stained for $1 \mathrm{~h}$. In order to visualize vancomycin, the fluorescent vancomycin BODIPY FL conjugate (ex488/em511) was added $(5 \mu \mathrm{g} / \mathrm{mL})$. Delafloxacin was visualized using the intrinsic fluorescence of the molecule (ex405/em450) at a concentration of $(10 \mu \mathrm{g} / \mathrm{mL})$. Concanavalin A (ex488/em545) was added $(50 \mu \mathrm{g} / \mathrm{mL})$ to visualize the biofilm matrix. The coverslip was mounted on the slides using Prolong Diamond Antifade (ThermoFisher) according to the manufactures recommendation. Biofilms were observed using a $60 \times$ oil immersion objective and an Olympus FV3000 laser scanning confocal microscope (Olympus, Tokyo, Japan). Images were acquired at a resolution of 512 by 512 pixels. To analyze the biofilms, a series of images at $\leq 1 \mu \mathrm{m}$ intervals in the $\mathrm{z}$ axis were acquired through the depth of the biofilm. For each condition, at least three fields of view were imaged and processed equally using cellSens Dimension Desktop V1.18 (Olympus). Representative images are displayed.

\section{Analysis of Matrix Coating and Antibiotic Accessibility Using Flow Cytometry}

To determine whether coating of $S$. aureus by $C$. albicans matrix components blocked antibiotic access to the cell we utilized vancomycin BODIPY FL and the intrinsic fluorescence of delafloxacin. Cultures were grown to mid-exponential phase, fluorescent compounds were added $10^{6} \mathrm{CFU} / \mathrm{mL}$ in $1 \% \mathrm{NaCl}$ at the same concentration that was used in the confocal experiments for $1 \mathrm{~h}$ at room temperature. Samples were analyzed using a Sony SH800 cell sorter.

\section{Concentrated Supernatant Time-Dependent Kill Assay}

Cultures $(25 \mathrm{~mL})$ of each strain (HG003 and SC5314) were grown in a shaking incubator overnight. These cultures were then pelleted and the supernatant removed. Supernatants were passed through a 0.45 micron filter then spun through a $3000 \mathrm{MW}$ filter and concentrated to approximately $1500 \mu \mathrm{L}$. Concentrated supernatant $(300 \mu \mathrm{L})$ was then added to planktonic HG003 cultures and incubated for $4 \mathrm{~h}$. These cultures were challenged with rifampicin $(0.8 \mu \mathrm{g} / \mathrm{mL})$. The bacteriostatic antibiotic, chloramphenicol $(4 \mu \mathrm{g} / \mathrm{mL})$, was added to prevent rifampicin resistant cells from regrowing. Aliquots $(100 \mu \mathrm{L})$ were removed from samples, and surviving bacteria were enumerated at 18, 24, 48 , and $72 \mathrm{~h}$ by serial dilution and plating on TSA.

\section{Farnesol Time-Dependent Kill Assay}

Overnight S. aureus cultures were diluted 1:1000 in TSB containing $40 \mu \mathrm{M}$ farnesol and grown to mid-exponential phase. Rifampicin $(0.8 \mu \mathrm{g} / \mathrm{mL})$ and chloramphenicol $(4 \mu \mathrm{g} / \mathrm{mL})$ were added and bacteria were enumerated over $72 \mathrm{~h}$.

\section{Spent Media Time-Dependent Kill Assay}

Overnight cultures were diluted 1:1000 and were grown to mid-exponential phase in $3 \mathrm{~mL}$ of either $\mathrm{HG} 003$ or SC5314 spent media collected from overnight cultures via centrifugation and challenged with rifampicin $(0.8 \mu \mathrm{g} / \mathrm{mL})$ and chloramphenicol $(4 \mu \mathrm{g} / \mathrm{mL})$. Bacteria were cultured and enumerated as described above.

\section{Determination of Intracellular ATP Concentration}

Intracellular ATP concentration was measured using the Promega BacTiter-Glo Microbial Cell Viability Assay according to manufacturer's instructions. Late exponential phase cultures were filtered through a $5 \mu \mathrm{M}$ filter to remove $C$. albicans. The remaining $S$. aureus cells were pelleted and washed with $1 \%$ $\mathrm{NaCl}$ prior to measuring luminescence. A sample was also taken for serial dilution and enumeration of bacteria. Luminescence was divided by surviving cells to account for any growth 
differences. Six replicates were used for obtaining averages and standard deviation. Significance was determined using a student's $t$-test, $P \leq 0.05$.

\section{Measurement of Membrane Potential in Individual Cells}

Membrane potential was measured using BacLight Bacterial Membrane Potential Kit according to manufacturer's instructions. Briefly, samples were taken from mid-exponential phase $(t=5 \mathrm{~h})$ in $S$. aureus either grown alone or in the presence of C. albicans. Samples were diluted to $1 \times 10^{6}$ cells in PBS and were stained with $\mathrm{DiOC}_{2}(3)$ for $30 \mathrm{~min}$ and analyzed by flow cytometry. Carbonyl cyanide m-chlorophenylhydrazone (CCCP) was used to dissipate membrane potential and was used to gate low membrane potential cells. Bacterial cells were separated from fungal cells and debris using back scatter (BSC) and forward scatter (FSC) parameters with 50,000 events collected for each sample. $\mathrm{DiOC}_{2}(3)$ was excited at $488 \mathrm{~nm}$ and emissions of the green and red fluorescence were detected with bandpass filters of 525/50- and 600/60-nm, respectively. Samples were analyzed using FlowJo software. The average and standard deviation was obtained from six biological replicates. Significance was determined using a student's $t$-test, $P \leq 0.05$.

\section{Quantifying Extracellular Glucose Availability}

Overnight cultures of $S$. aureus (1:1000) and C. albicans (1:100) were diluted in TSB and placed in a shaking incubator. Every hour, $500 \mathrm{uL}$ media was removed and pelleted. Supernatant was then used to measure glucose concentration using an Invitrogen glucose detection colorimetric assay kit according to manufacturer's instructions. Averages and standard deviation were calculated using six biological replicates.

\section{Measuring Fumarase $\mathbf{C}$ Expression}

Overnight cultures of $S$. aureus (1:100) containing PfumC:gfp or Pspa:gfp plasmids and C. albicans (1:50) were diluted in Mueller Hinton Broth (MHB) in a microtiter plate. Growth and fluorescence were $(485 \mathrm{ex} / 528 \mathrm{em})$ were monitored over $22 \mathrm{~h}$ in a Biotek microplate reader at $37^{\circ} \mathrm{C}$ with continuous shaking. Averages and standard deviation were calculated from biological triplicates.

\section{RESULTS}

\section{Polymicrobial Cultures Demonstrate Increased Tolerance to Antibiotics in Both Biofilm and Planktonic Environments}

Polymicrobial infections are more tolerant to antibiotic therapy than single organism infections, though the underlying mechanisms remain unclear. Recent work has demonstrated that the presence of $C$. albicans increases $S$. aureus tolerance to vancomycin within a biofilm (Kong et al., 2016). We sought to determine if interactions between $C$. albicans and S. aureus lead to multidrug tolerance. Since mature biofilms often do not respond to antibiotics, it is often impossible to observe decreased antibiotic effectiveness between various cultures. In order to overcome this, immature biofilms were used. It is important to note that two distinct phenotypes of the wild type HG003 strain were observed. Following antibiotic treatment, cultures showed up to 3 logs of killing, or little to no effect. Polymicrobial biofilms led to significantly more survival in six of eight antibiotic treatments (ciprofloxacin $p=0.006$, oxacillin $p=0.019$, rifampicin $p=0.006$, rifampicin/ciprofloxacin $p=0.001$, rifampicin/gentamicin $p=0.003$, and vancomycin/ciprofloxacin $p=0.008$ ) compared to $S$. aureus monomicrobial biofilms (Figure 1). Interestingly, no increase in tolerance was observed when biofilms were challenged with vancomycin.

To determine if the increase in tolerance was specific to biofilms, planktonic cultures were challenged with antibiotics during the mid-exponential growth phase. Following antibiotic challenge with rifampicin, ciprofloxacin, oxacillin, delafloxacin, and vancomycin, S. aureus exhibited 10 to 100 -fold more persisters when grown in the presence of $C$. albicans (Figure 2). To further determine these effects were not strain or species specific, another MSSA strain, a MRSA strain, and a $S$. epidermidis strain were tested for antibiotic tolerance in the presence and absence of $C$. albicans (Supplementary Figures S1A-C). With all three strains, there was increased antibiotic tolerance when the staphylococcal species was grown in the presence of C. albicans. These experiments demonstrate the presence of $C$. albicans increases $S$. aureus persister cells

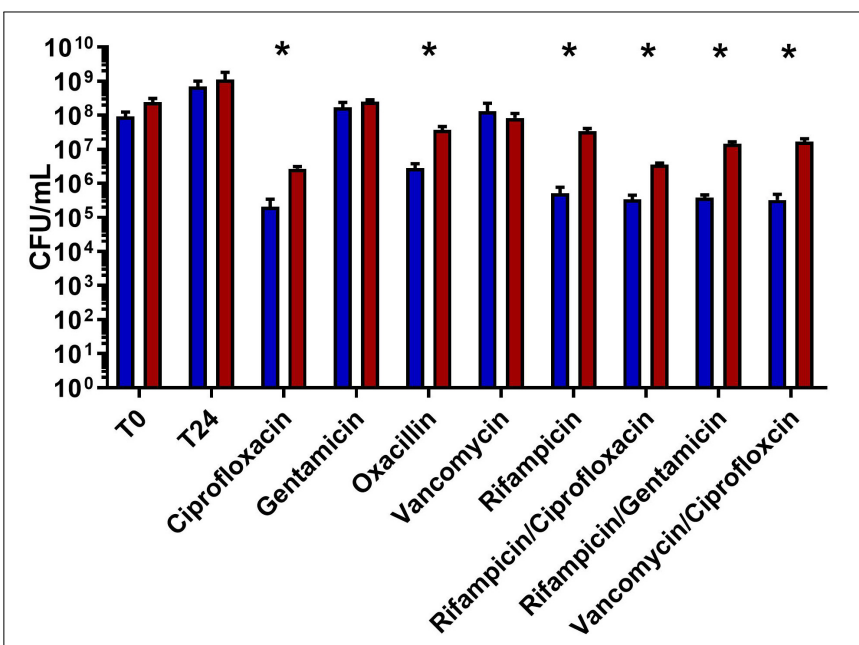

FIGURE 1 | Polymicrobial biofilms show increased tolerance to a variety of antibiotic. Overnight cultures of $S$. aureus were diluted 1:1000 and C. albicans overnight cultures were diluted 1:100 in TSB using a microtiter plate. Plates were incubated for $8 \mathrm{~h}$ at $37^{\circ} \mathrm{C}$ statically. Non-adherent cells washed, fresh media was added, and biofilms were subsequently challenged with antibiotics (10-100 $\times$ MIC) for 24 h. S. aureus growing in polymicrobial biofilms (red) had significantly higher survival compared to biofilms only containing $S$. aureus (blue). Experiment was performed in biological triplicate and error bars represent standard deviation. Significance (as indicated by *) was determined using a $t$-test $(p<0.05)$. 

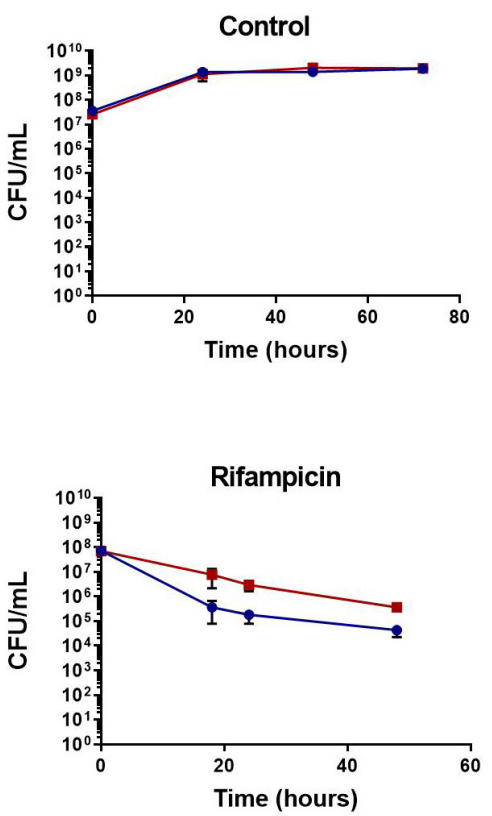
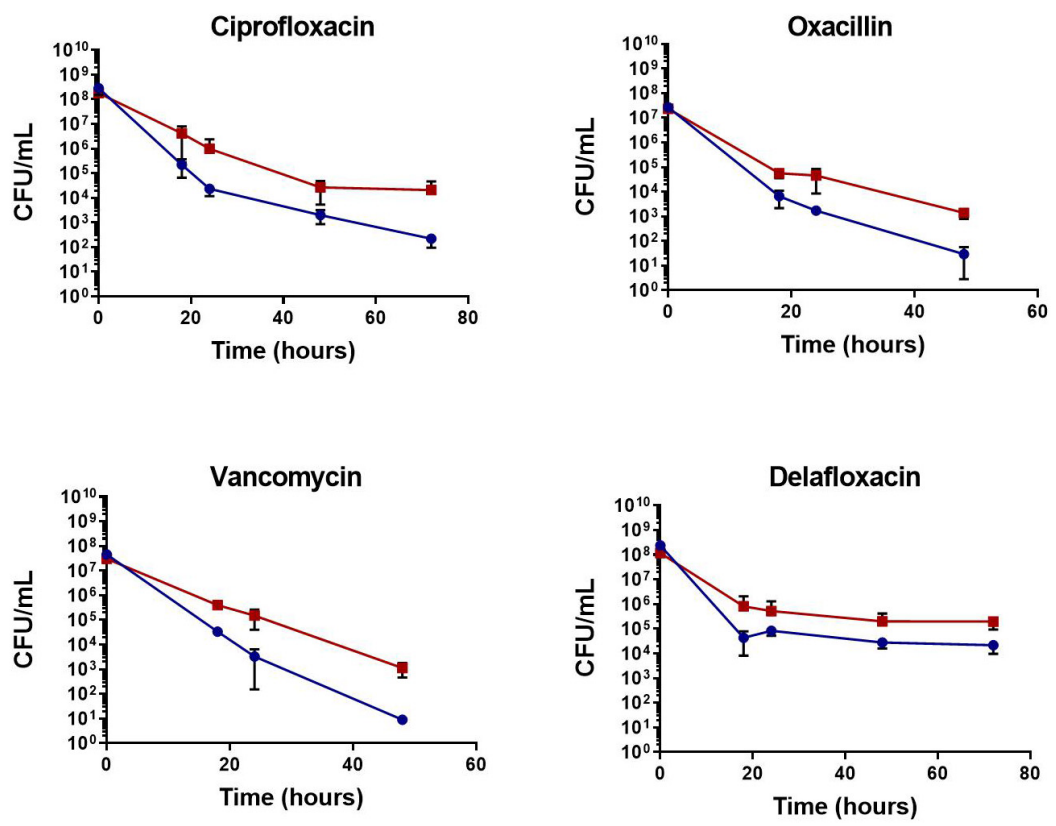

FIGURE 2 | Polymicrobial planktonic cultures have increased antibiotic tolerance. Planktonic cultures were grown to mid-exponential phase in TSB and challenged with antibiotic (10-100 $\times$ MIC), the surviving bacteria were enumerated over 48 or $72 \mathrm{~h}$ by plating on TSA containing amphotericin B ( $25 \mu \mathrm{g} / \mathrm{mL})$. The presence of C. albicans increases $S$. aureus (red) antibiotic tolerance compared to $S$. aureus monocultures (blue). Experiment was performed in biological triplicate and error bars represent standard deviation.

when challenged with most antibiotics, regardless of whether growing in planktonic or biofilm environments.

\section{With the Exception of Vancomycin, Antibiotics Diffuse Freely Through Polymicrobial Biofilms}

One mechanism that could explain the increased tolerance in polymicrobial biofilms is that antibiotics are not able to completely penetrate the polymicrobial biofilm matrix. To determine whether this was the case for other classes of antibiotics, antibiotic penetration assays were performed. The respective zone of inhibition for oxacillin, rifampicin, delafloxacin, and ciprofloxacin indicated that these antibiotics are not impeded by the biofilm matrix created by $S$. aureus, C. albicans, or the combination of both organisms (Table 1).
As previously demonstrated, vancomycin diffusion was inhibited by the polymicrobial biofilm $(p=0.041)$. A decrease in vancomycin diffusion was also seen with $S$. aureus monoculture biofilms, although this was found to not be significantly different ( $p=0.052$ ) from diffusion in the absence of biofilm.

To confirm these findings, vancomycin and delafloxacin penetration throughout the biofilm were examined using confocal scanning laser microscopy (Figure 3). S. aureus, C. albicans, or polymicrobial biofilms were grown. Formed biofilms were visualized by staining the polysaccharide matrix with concanavalin A (ConA, red). To visualize vancomycin, a fluorescent BODIPY conjugate was used (green). For delafloxacin, its intrinsic fluorescence was used (ex405/em450, blue). Contrary to the biofilm penetration assay and previously published work, vancomycin diffusion did not appear to be inhibited by the polymicrobial biofilm. The only exception to this

TABLE 1 | Zone of inhibition (mm) following antibiotic diffusion through biofilms.

\begin{tabular}{lcccc}
\hline & \multicolumn{3}{c}{ Zone of Inhibition (MM) } \\
\hline Antibiotic & No biofilm & S. aureus biofilm & C. albicans biofilm & Polymicrobial biofilm \\
\hline Ciprofloxacin & $76.67 \pm 5.77$ & $78.33 \pm 7.63$ & $75 \pm 8.66$ & $76.67 \pm 5.77$ \\
Delafloxacin & $160 \pm 5$ & $150 \pm 5$ & $150 \pm 0$ & $151.67 \pm 2.89$ \\
Oxacillin & $120 \pm 17.32$ & $123.33 \pm 15.27$ & $96.67 \pm 2.89$ & 18.03 \\
Rifampicin & $100 \pm 5$ & $95 \pm 5$ & $35 \pm 0$ & $96.67 \pm 5.77$ \\
Vancomycin & $40 \pm 5$ & $13.33 \pm 12.58$ & & $13.33 \pm 11.55^{*}$ \\
No antibiotic & $0 \pm 0$ & & & \\
\hline
\end{tabular}

*Denotes significance using $t$-test $(p \leq 0.05)$. 


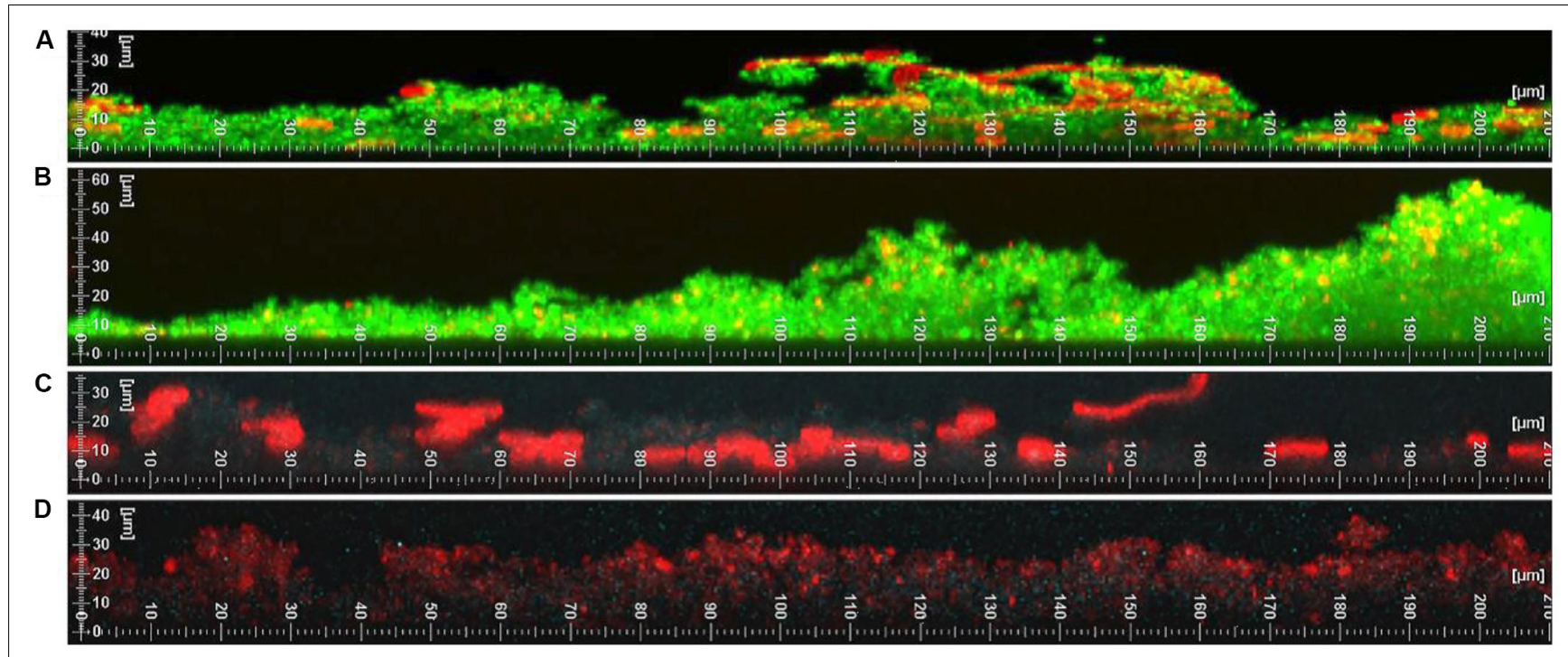

FIGURE 3 | Vancomycin has limited diffusion while Delafloxacin does not have restricted diffusion through polymicrobial biofilms. (A) Vancomycin BODIPY FL conjugate (green) and concanavalin A (red) was added to a polymicrobial biofilm. The diffusion of vancomycin is modestly reduced in areas with a complex and thick biofilm. (B) Vancomycin BODIPY FL conjugate (green) and concanavalin A (red) was added to a monomicrobial S. aureus biofilm. The S. aureus biofilm has unrestricted diffusion of vancomycin. (C) Delafloxacin (blue) and concanavalin A (red) was added to a polymicrobial biofilm. The diffusion of delafloxacin is not restricted throughout the biofilm. (D) Delafloxacin (blue) and concanavalin A (red) was added to a monomicrobial S. aureus biofilm. The S. aureus biofilm has unrestricted diffusion of delafloxacin. Images are respective images from three random fields of view.

observation is a slight decrease in vancomycin fluorescence in basal layers of the biofilm that reached $30 \mu \mathrm{M}$ in height. However, a similar decrease in fluorescence was observed in biofilms formed by $S$. aureus alone. Despite this very modest phenotype, vancomycin was able to diffuse throughout the biofilm and reach all of the cells growing within the biofilm. Similarly, although the intrinsic fluorescence only produced a weak signal, delafloxacin was not inhibited by either single or polymicrobial biofilms. With the possible exception of vancomycin, the increased tolerance does not appear to be due to limited penetration of the antibiotic through the biofilm matrix.

\section{Vancomycin Binding in Planktonic Cultures Is Not Inhibited by Matrix Coating}

Confocal imaging revealed ConA binding S. aureus within polymicrobial cultures. To determine whether this coating was enough to inhibit antibiotics from accessing the cell, flow cytometry was used to measure the amount of antibiotics able to bind to the bacteria. Vancomycin was found to bind similarly to $S$. aureus cells regardless of whether they were grown in monomicrobial cultures or polymicrobial cultures (Figure 4). To confirm that the growth to mid-exponential phase was long enough for matrix coating to occur, polymicrobial cultures were stained with ConA. Matrix coating did occur during this time as indicated by the fluorescence associated with $S$. aureus cells in polymicrobial cultures. Unfortunately, the intrinsic fluorescence of delafloxacin was too weak for analysis and could not be properly assessed. Nevertheless, it is clear that despite coating of bacterial cells, vancomycin was still able to bind to S. aureus, and physical inhibition of the antibiotic is not the reason for increased tolerance.

\section{Increased Antibiotic Tolerance Within S. aureus and C. albicans Co-cultures Is Not Affected by Secreted Products}

Secreted C. albicans products larger than 3,000 MW were concentrated and added to cultures prior to antibiotic challenge to examine whether a specific virulence factor or biomolecule was influencing tolerance within $S$. aureus. After an incubation period, cultures were challenged with rifampicin. Cultures containing concentrated supernatant showed no difference in antibiotic tolerance compared to cultures incubated without the added supernatant (Figure 5A).

Previously, farnesol was shown to influence antibiotic tolerance (Jabra-Rizk et al., 2006; Kong et al., 2017). According to recent work, at high concentrations (100-150 $\mu \mathrm{M})$, farnesol appears to enhance antibiotic effectiveness. Conversely, lower concentrations $(40 \mu \mathrm{M})$ of farnesol appear to result in increased antibiotic tolerance. Therefore, we tested the possibility that increased tolerance is from farnesol secretion by C. albicans. Following the addition of farnesol $(40 \mu \mathrm{M})$, no effect on antibiotic tolerance was observed when cultures were challenged with rifampicin (Figure 5B).

We considered the possibility that secreted products smaller than 3,000 MW were being excluded from these kill assays. To confirm previous findings, cultures were grown in spent media prior to antibiotic challenge. Growth in spent C. albicans media increased tolerance within $S$. aureus, however, growth in spent $S$. aureus media also increased tolerance to a similar extent 

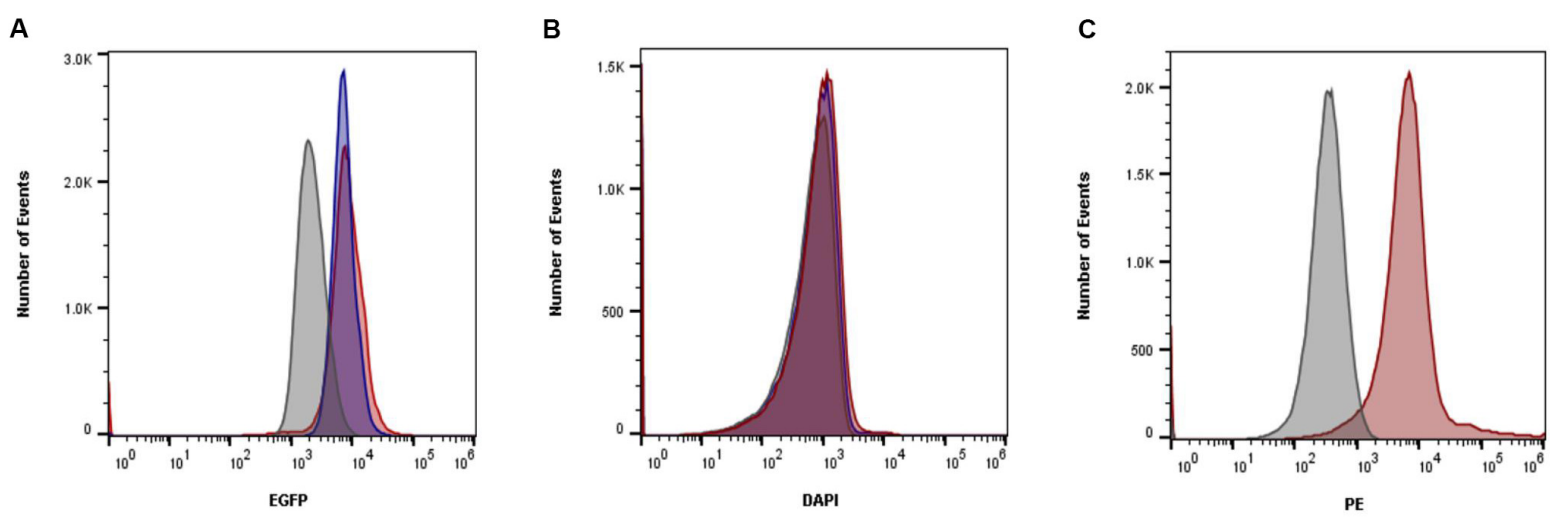

FIGURE 4 | Matrix coating does not inhibit vancomycin binding. (A) Vancomycin BODIPY FL conjugate was added to planktonic cells in either polymicrobial (red) or monomicrobial (blue) cultures. Vancomycin was able to bind S. aureus similarly in both conditions. Unstained cells were included as a control (gray).

(B) Fluorescence from delafloxacin either in polymicrobial (red) or monomicrobial (blue) cultures was unable to be differentiated from unstained cells (gray) due to the weak signal produced. (C) To ensure matrix coating occurred, ConA was added to polymicrobial (red) cultures and compared to unstained polymicrobial cultures (gray). Data is representative of three independent replicates.
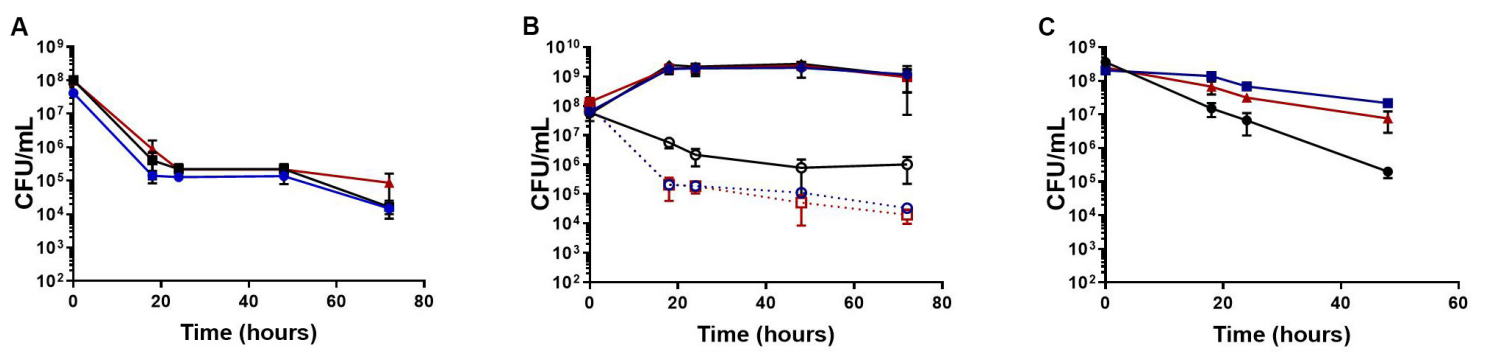

FIGURE 5 | Products secreted by C. albicans are not responsible for increased tolerance. (A) Planktonic cultures were grown to mid-exponential phase in TSB (blue) with the addition of concentrated C. albicans (red) or S. aureus (black) secreted products larger than $3000 \mathrm{MW}$ and subsequently challenged with rifampicin. No change in tolerance was observed when compared to the control. (B) HGOO3 (solid blue line) showed no growth defect in the presence of farnesol (black solid line) or in the presence of $C$. albicans (solid red line). The presence of farnesol did not increase tolerance to rifampicin (dashed black) compared to $S$. aureus in monomicrobial cultures (dashed blue line). S. aureus grown in the presence of $C$. albicans (dashed red line) increased survival by a log. (C) Planktonic $S$. aureus cultures were grown to mid-exponential phase in TSB (black), in spent $S$. aureus media (blue), or in spent $C$. albicans media (red). Cultures were challenged with rifampicin and surviving bacteria were enumerated over $48 \mathrm{~h}$. Growth in both S. aureus and C. albicans spent supernatant increased survival following antibiotic challenge. All experiments were performed in biological triplicate and error bars represent standard deviation.

(Figure 5C). These results cast doubt on the ability of secreted C. albicans products to increase antibiotic tolerance. Instead, the increase in tolerance in both environments suggests that a common cause, such as nutrient depletion, is responsible for increased tolerance.

\section{Polymicrobial Cultures Consume Glucose at an Increased Rate, Leading to Lower Intracellular ATP Concentrations}

Glucose, a preferred source of carbon for S. aureus, serves as the major substrate for glycolysis. This leads to NADH generation and subsequent ATP synthesis. Glucose concentration was measured over time to determine if a polymicrobial culture could deplete available glucose at an increased rate. As one would expect, glucose was consumed faster in the polymicrobial culture than the S. aureus monoculture (Figure 6).

To confirm that the lower concentrations of extracellular glucose affect the energy status of bacterial cells, the intracellular
ATP in S. aureus from single and mixed cultures was measured. Previous work demonstrated that antibiotic tolerance is increased when intracellular ATP is depleted (Conlon et al., 2016). During late exponential phase, $S$. aureus cells from mixed cultures exhibited lower intracellular ATP concentrations compared to S. aureus from single cultures (Figure 7). Moreover, membrane potential is closely linked with the energy status of the cell, and therefore it is likely altered in polymicrobial cultures. S. aureus cells grown in the presence of $C$. albicans exhibited a reduced membrane potential compared to $S$. aureus monocultures (Figure 8). This indicates that polymicrobial cultures consume nutrients more rapidly than monomicrobial cultures, resulting in lower intracellular ATP and membrane potential.

\section{Cells in Polymicrobial Biofilms Show a Decrease in Metabolic Gene Activity}

Recent work has implicated an association between the TCA cycle and membrane potential and persister cell formation in 


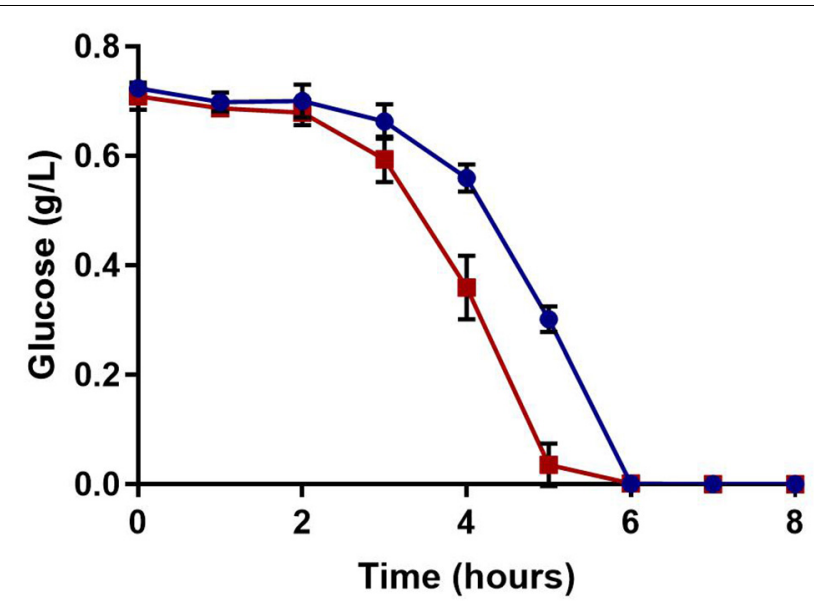

FIGURE 6 | Extracellular glucose availability. Glucose concentrations were measured over time. Glucose was more rapidly consumed in polymicrobial cultures (red) compared to $S$. aureus monocultures (blue); both cultures completely exhausted the glucose in the media by $6 \mathrm{~h}$. Experiments were performed in triplicate and error bars represent standard deviation.

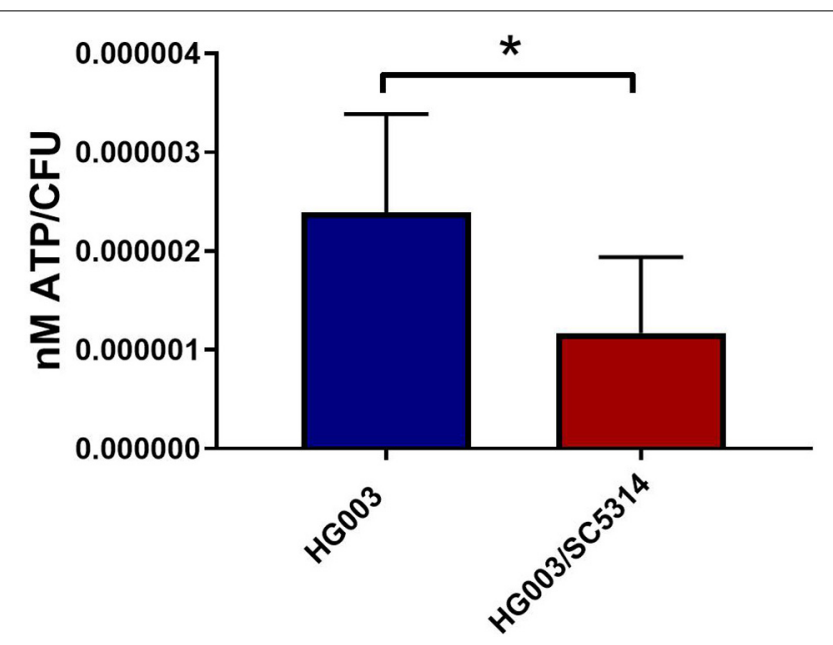

FIGURE 7 | Staphylococcus aureus grown in polymicrobial cultures has lower intracellular ATP. Planktonic cultures were grown to late exponential phase, pelleted and washed, and intracellular ATP was measured. Bacterial numbers were determined by standard serial dilution technique and ATP concentrations were normalized to CFU. Data is represented by the mean of six independent replicates and error bars represent standard deviation. Significance was determined using a $t$-test $\left({ }^{*} p<0.05\right)$.

S. aureus (Wang et al., 2018; Zalis et al., 2019). To examine whether a similar mechanism was occurring in polymicrobial cultures, TCA cycle activity was measured using a promoter- $g f p$ fusion construct, PfumC:gfp. In the presence of C. albicans, fluorescence was notably lower over a period of $22 \mathrm{~h}$ (Figure 9). In order to assess if this effect was from a generalized reduction in transcription or specific to genes in central metabolism, Pspa:gfp was used as a control reporter. The spa gene encodes the virulence factor, protein A. The spa reporter had no difference between $S$. aureus cells grown alone compared to those cells grown in a polymicrobial culture, thus indicating decreased transcription was specific to metabolic processes.

\section{DISCUSSION}

It is estimated that fifty percent of all infections involve biofilms (Wolcott et al., 2010). Biofilm infections are notoriously difficult to eradicate completely, despite being caused primarily by drug-susceptible pathogens (Monack et al., 2004; Lewis, 2010; Conlon, 2014). Further complications arise when biofilms involve more than one organism, resulting in increased mortality (Gabrilska and Rumbaugh, 2015). Reasons for this increased mortality remain unclear but a number of studies have focused on individual antibiotic treatment as well as specific reasons for therapy failure. Increased antimicrobial resistance has been observed for a limited number of antibiotics (Harriott and Noverr, 2009), but this fails to explain recurring infections caused by drug-susceptible organisms. Our data provide an explanation for multi-drug tolerance by a broad acting energy-dependent mechanism. This is in accordance with recent work published on the mechanism of persister formation in S. aureus (Conlon et al., 2016; Wang et al., 2018; Zalis et al., 2019).

Polymicrobial biofilms were consistently more tolerant to antibiotics with the exception of vancomycin and gentamicin. This contradicts other findings, where the presence of C. albicans increased tolerance to both of these antibiotics (Singh et al., 2010; Perez et al., 2014; Li et al., 2015; Kong et al., 2016). This does not mean that there is no difference, and may be a result of little to no killing observed in either the monomicrobial or polymicrobial biofilms challenged with these antibiotics. Higher concentrations of antibiotics may show results similar to previously published work. However, a more interesting phenomena demonstrated here is that antibiotic diffusion through the biofilm did not appear to be a significant cause of increased antibiotic tolerance within the biofilm. In most cases, there was no significant difference between the zone of inhibition following diffusion through a polymicrobial or monomicrobial biofilm. Vancomycin diffusion was variable between replicates with one assay exhibiting no diffusion and the other replicates having impeded diffusion. The possibility exists that vancomycin is simply defusing through the biofilm at a slower rate than the other antibiotics. Confocal analysis also provided evidence that delafloxacin was not impeded by biofilm matrix. While the intrinsic fluorescent signal was faint, it is clearly present in the deeper biofilm layers.

Further support that physical inhibition is not the primary mechanism for increased tolerance is provided by experiments performed in a planktonic setting. It could be assumed that if physical inhibition was the primary mechanism for increased tolerance, there would be little difference in tolerance to non-cell wall acting antibiotics in a planktonic environment. However, the large increases in tolerance were consistent across all classes of antibiotics used, indicating that physical inhibition is not a likely explanation for multidrug tolerance. Further evidence against physical inhibition was provided by flow cytometry analysis. While the fluorescence from delafloxacin was too weak to be 
A

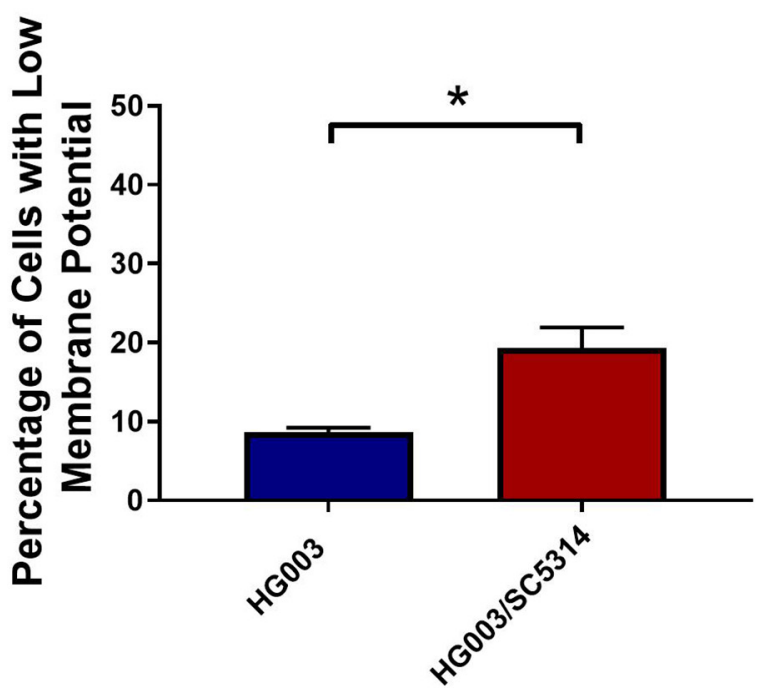

B

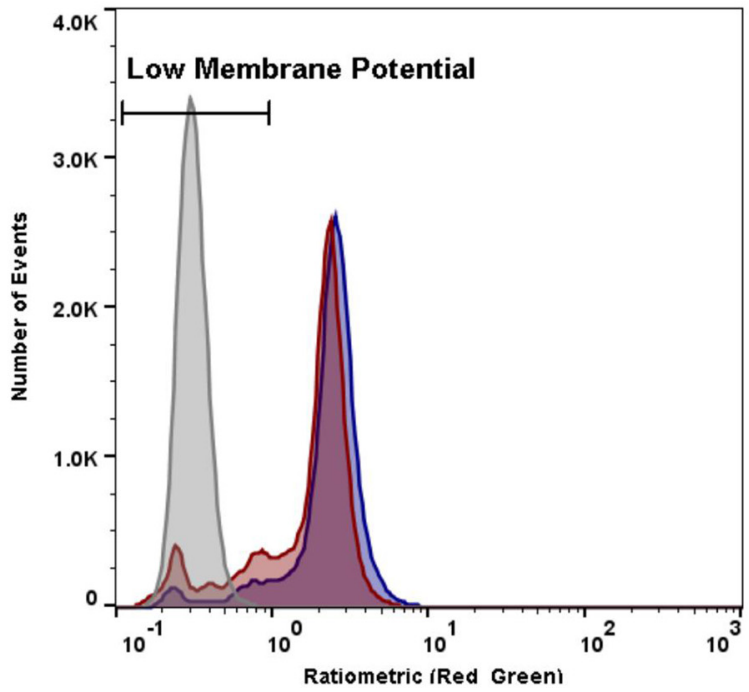

FIGURE 8 | The presence of C. albicans decreases membrane potential in S. aureus cells. (A) Membrane potential was measured during mid-exponential phase in S. aureus either grown alone (blue) or in the presence of $C$. albicans (red). $1 \times 10^{6}$ cells in PBS were stained with DiOC $2(3)$ for 30 min and analyzed by flow cytometry. (B) Carbonyl cyanide $m$-chlorophenylhydrazone (CCCP) was used to dissipate membrane potential (gray) and was used to gate low membrane potential cells. The mean \pm SD is shown, $n=6$ for the graph on the left. The figure on the right is representative of six independent replicates. Significance was determined using a $t$-test $\left({ }^{*} p<0.05\right)$.
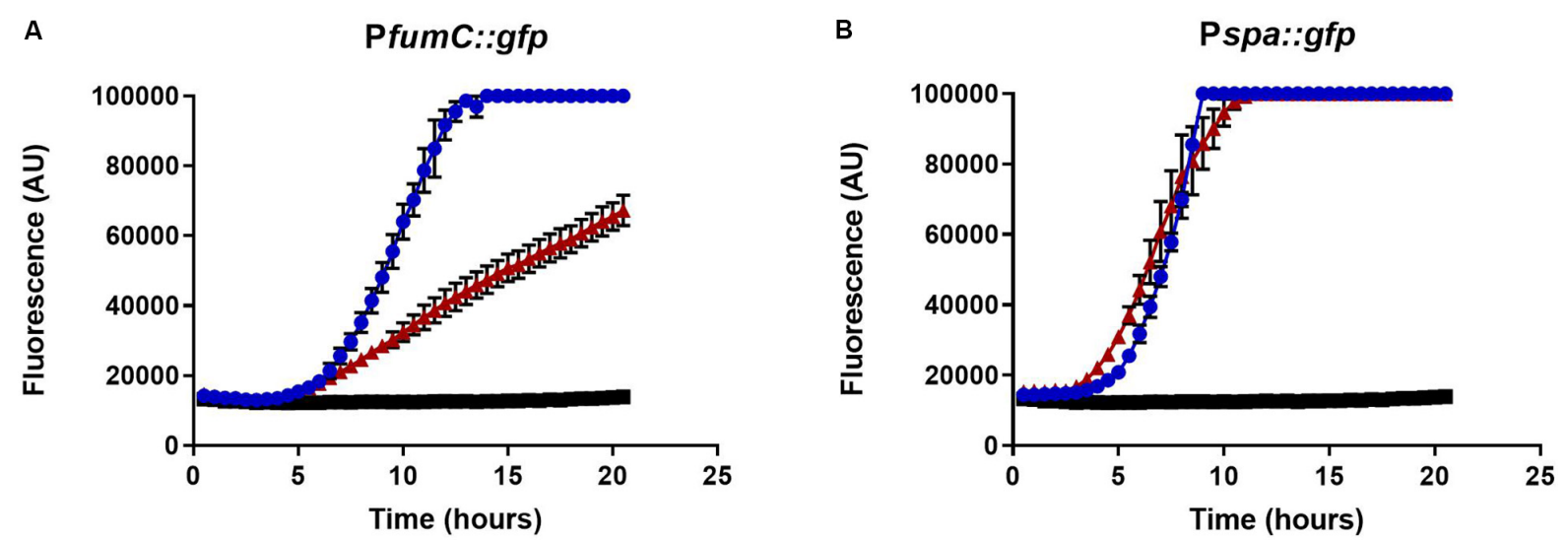

FIGURE 9 | Fumarase C Expression. GFP expression of PfumC:gfp (A) and Pspa:gfp (B) was measured over time using a Biotek microplate reader. Overnight cultures of $S$. aureus $(1: 100)$ and C. albicans (1:50) were diluted in MHB in a microtiter plate. C. albicans decreased expression of the TCA cycle gene, fumarase (red) compared to expression observed when $S$. aureus was grown alone (blue). This effect was specific to fumarase and not the result of a generalized reduction in S. aureus transcription as indicated by the Pspa:gfp control. C. albicans did not affect fluorescence outside of gene expression (black). Experiments were performed in biological triplicate; error bars represent standard deviation.

detected with our flow cytometer, vancomycin was clearly not inhibited by matrix coating from C. albicans.

Previous work has found the $C$. albicans quorum sensing molecule, farnesol, may both increase and decrease antibiotic susceptibility depending on its concentration (Jabra-Rizk et al., 2006; Kong et al., 2017). The effects of secreted products, including farnesol, on antibiotic tolerance were tested. Neither concentrated $C$. albicans nor $S$. aureus supernatant affected tolerance, indicating that extracellular byproducts larger than $3000 \mathrm{MW}$ are not influencing antibiotic tolerance in S. aureus.
However, this still leaves the possibility of smaller molecules influencing the bacteria.

Small products other than farnesol were further investigated by growing cultures in the presence of spent media. Growth in C. albicans conditioned media did increase antibiotic tolerance, however, the same phenotype was observed when grown in spent $S$. aureus media. Unexpectedly, the increase on antibiotic tolerance does not appear to be specific to a product secreted by $C$. albicans, rather, nutrient exhaustion was a more likely explanation for the observed increase in antibiotic tolerance. 
Recent work on the mechanism of persister formation has implicated decreased intracellular ATP and membrane potential with an increase in antibiotic tolerance (Conlon et al., 2016; Shan et al., 2017; Wang et al., 2018; Zalis et al., 2019). Results from the spent media assay suggest that the increased tolerance in polymicrobial cultures can be explained by a similar mechanism. It follows that if $C$. albicans is decreasing available nutrients within the biofilm, $S$. aureus cells will have to compete for the same nutrients. Those cells, which are unable to find adequate nutrients will create a population of $S$. aureus cells in a low energy state, leading to an increase in tolerance to antibiotics with active targets. Available glucose was depleted faster in polymicrobial cultures and, fittingly, both ATP and membrane potential were lower in $S$. aureus cells grown in a mixed culture compared to monocultures. A specific mechanism with the TCA cycle was recently suggested (Wang et al., 2018; Zalis et al., 2019), and results with the TCA cycle reporter, PfumC:gfp, support those observations. Together these results demonstrate a decrease in $S$. aureus metabolism as a direct result of nutrient depletion by C. albicans.

Metabolism is becoming a focal point in the investigation of chronic S. aureus infections. While glycolysis is required for initial abscess formation in mice, upon maturation of the abscess glucose concentrations become a limiting factor (Richardson et al., 2015; Vitko et al., 2015; Thurlow et al., 2018). Similarly, during initial stages of biofilm formation, glucose is likely readily available and preferentially consumed. Later on in the process, the biofilm becomes a glucose-limited environment before subsequent dispersal of the biofilm (Boles and Horswill, 2008; Huynh et al., 2012). These examples are niches where antibiotic treatment of $S$. aureus is likely to fail. Furthermore, these niches often lead to chronic infections that the immune system is unable to manage (Leid et al., 2002; Jesaitis et al., 2003; Vuong et al., 2004; Cheng et al., 2011). Nutrient depletion leading to an antibiotic tolerant state may hold broader implications with parallels in chronic infections with other microorganisms.

\section{DATA AVAILABILITY STATEMENT}

All datasets generated for this study are included in the article/Supplementary Material.

\section{REFERENCES}

Amato, S. M., and Brynildsen, M. P. (2014). Nutrient transitions are a source of persisters in Escherichia coli biofilms. PLoS One 9:e93110. doi: 10.1371/journal. pone.0093110

Boles, B. R., and Horswill, A. R. (2008). Agr-mediated dispersal of Staphylococcus aureus biofilms. PLoS Pathog. 4:e1000052. doi: 10.1371/journal.ppat.1000052

Cheng, A. G., DeDent, A. C., Schneewind, O., and Missiakas, D. (2011). A play in four acts: Staphylococcus aureus abscess formation. Trends Microbiol. 19, 225-232. doi: 10.1016/j.tim.2011.01.007

Cheung, A. L., Nast, C. C., and Bayer, A. S. (1998). Selective activation of sar promoters with the use of green fluorescent protein transcriptional fusions as the detection system in the rabbit endocarditis model. Infect. Immun. 66, 5988-5993.

\section{AUTHOR CONTRIBUTIONS}

$\mathrm{DN}, \mathrm{BL}$, and $\mathrm{AN}$ contributed to the conception and design of the study. DN and AN performed the statistical analysis. DN wrote the first draft of the manuscript. DN, SS, BL, and AN wrote sections of the manuscript. All authors performed the experiments, generated data appearing in the manuscript, contributed to the revision of the manuscript, and read and approved the submitted version.

\section{FUNDING}

This work was funded by National Center for Research Resources (5P20RR016469) and the National Institute for General Medical Science (8P20GM103427). Funding for this work was also provided by the Nebraska EPSCoR Undergraduate Research Experience at Small Colleges and Universities program and the Nebraska Research Initiative for equipment used in this project. Funding for the open access publication fees were provided by UNK Biology Department.

\section{ACKNOWLEDGMENTS}

We would like to thank Kim Lewis, Northeastern University, for the PfumC:gfp and Pspa:gfp plasmids.

\section{SUPPLEMENTARY MATERIAL}

The Supplementary Material for this article can be found online at: https://www.frontiersin.org/articles/10.3389/fmicb. 2019.02803/full\#supplementary-material

FIGURE S1 | Increased antibiotic tolerance associated with polymicrobial cultures are not strain or species specific. Planktonic cultures were grown to early to mid-exponential phase in TSB and challenged with vancomycin $(100 \times \mathrm{MIC})$, the surviving bacteria were enumerated over $72 \mathrm{~h}$ by plating on TSA containing amphotericin B $(25 \mu \mathrm{g} / \mathrm{mL})$. The presence of C. albicans increases $S$. aureus UAMS-1 (A), S. aureus JE2 (B), and S. epidermidis 1457 (C) (red) antibiotic tolerance compared to $S$. aureus monocultures (blue). Experiment was performed in biological triplicate and error bars represent standard deviation.

Conlon, B. P. (2014). Staphylococcus aureus chronic and relapsing infections: evidence of a role for persister cells: an investigation of persister cells, their formation and their role in S. aureus disease. Bioessays 36, 991-996. doi: 10. 1002/bies.201400080

Conlon, B. P., Rowe, S. E., Gandt, A. B., Nuxoll, A. S., Donegan, N. P., Zalis, E. A., et al. (2016). Persister formation in Staphylococcus aureus is associated with ATP depletion. Nat. Microbiol. 1:16051. doi: 10.1038/nmicrobiol.2016.51

Ericson, J. E., Popoola, V. O., Smith, P. B., Benjamin, D. K., Fowler, V. G., Benjamin, D. K. Jr., et al. (2015). Burden of invasive Staphylococcus aureus infections in hospitalized infants. JAMA Pediatr. 169, 1105-1111. doi: 10.1001/ jamapediatrics.2015.2380

Fengcai, S., Di, X., Qianpeng, H., Hongke, Z., and Yiyu, D. (2015). [Microbial characteristics in culture-positive sepsis and risk factors of polymicrobial infection in ICU]. Zhonghua Wei Zhong Bing Ji Jiu Yi Xue 27, 718-723. 
Gabrilska, R. A., and Rumbaugh, K. P. (2015). Biofilm models of polymicrobial infection. Future Microbiol. 10, 1997-2015. doi: 10.2217/fmb.15.109

Gillum, A. M., Tsay, E. Y., and Kirsch, D. R. (1984). Isolation of the Candida albicans gene for orotidine- $5^{\prime}$-phosphate decarboxylase by complementation of S. cerevisiae ura3 and E. coli pyrF mutations. Mol. Gen. Genet. 198, 179-182. doi: $10.1007 / \mathrm{bf} 00328721$

Goetghebeur, M., Landry, P. A., Han, D., and Vicente, C. (2007). Methicillinresistant Staphylococcus aureus: a public health issue with economic consequences. Can. J. Infect. Dis. Med. Microbiol. 18, 27-34.

Haley, K. P., and Skaar, E. P. (2012). A battle for iron: host sequestration and Staphylococcus aureus acquisition. Microbes Infect. 14, 217-227. doi: 10.1016/ j.micinf.2011.11.001

Halsey, C. R., Lei, S., Wax, J., Lehman, M. K., Nuxoll, A. S., Steinke, L., et al. (2016). Amino acid catabolism in Staphylococcus aureus and the function of carbon catabolite repression. mBio 8:e1434-16.

Harriott, M. M., and Noverr, M. C. (2009). Candida albicans and Staphylococcus aureus form polymicrobial biofilms: effects on antimicrobial resistance. Antimicrob. Agents Chemother. 53, 3914-3922. doi: 10.1128/AAC.00657-09

Harriott, M. M., and Noverr, M. C. (2010). Ability of Candida albicans mutants to induce Staphylococcus aureus vancomycin resistance during polymicrobial biofilm formation. Antimicrob. Agents Chemother. 54, 3746-3755. doi: 10.1128/ AAC.00573-10

Harriott, M. M., and Noverr, M. C. (2011). Importance of Candida-bacterial polymicrobial biofilms in disease. Trends Microbiol. 19, 557-563. doi: 10.1016/ j.tim.2011.07.004

Hassoun, A., Linden, P. K., and Friedman, B. (2017). Incidence, prevalence, and management of MRSA bacteremia across patient populations-a review of recent developments in MRSA management and treatment. Crit. Care 21:211. doi: 10.1186/s13054-017-1801-3

Herbert, S., Ziebandt, A. K., Ohlsen, K., Schafer, T., Hecker, M., Albrecht, D., et al. (2010). Repair of global regulators in Staphylococcus aureus 8325 and comparative analysis with other clinical isolates. Infect. Immun. 78, 2877-2889. doi: 10.1128/IAI.00088-10

Huynh, T. T., McDougald, D., Klebensberger, J., Al Qarni, B., Barraud, N., Rice, S. A., et al. (2012). Glucose starvation-induced dispersal of Pseudomonas aeruginosa biofilms is cAMP and energy dependent. PLoS One 7:e42874. doi: 10.1371/journal.pone.0042874

Jabra-Rizk, M. A., Meiller, T. F., James, C. E., and Shirtliff, M. E. (2006). Effect of farnesol on Staphylococcus aureus biofilm formation and antimicrobial susceptibility. Antimicrob. Agents Chemother. 50, 1463-1469. doi: 10.1128/ AAC.50.4.1463-1469.2006

Jesaitis, A. J., Franklin, M. J., Berglund, D., Sasaki, M., Lord, C. I., Bleazard, J. B., et al. (2003). Compromised host defense on Pseudomonas aeruginosa biofilms: characterization of neutrophil and biofilm interactions. J. Immunol. 171, 4329-4339. doi: 10.4049/jimmunol.171.8.4329

Kelly, B., and O'Neill, L. A. (2015). Metabolic reprogramming in macrophages and dendritic cells in innate immunity. Cell Res. 25, 771-784. doi: 10.1038/cr.2015. 68

Koch, A. M., Nilsen, R. M., Dalheim, A., Cox, R. J., and Harthug, S. (2015a). Need for more targeted measures - only less severe hospital-associated infections declined after introduction of an infection control program. J. Infect. Public Health 8, 282-290. doi: 10.1016/j.jiph.2014.11.001

Koch, A. M., Nilsen, R. M., Eriksen, H. M., Cox, R. J., and Harthug, S. (2015b). Mortality related to hospital-associated infections in a tertiary hospital; repeated cross-sectional studies between 2004-2011. Antimicrob. Resist. Infect. Control 4:57. doi: 10.1186/s13756-015-0097-9

Kong, E. F., Tsui, C., Kucharikova, S., Andes, D., Van Dijck, P., and JabraRizk, M. A. (2016). Commensal protection of Staphylococcus aureus against antimicrobials by Candida albicans biofilm matrix. $m$ Bio 7:e1365-16. doi: 10 . 1128/mBio.01365-16

Kong, E. F., Tsui, C., Kucharikova, S., Van Dijck, P., and Jabra-Rizk, M. A. (2017). Modulation of Staphylococcus aureus response to antimicrobials by the Candida albicans quorum sensing molecule farnesol. Antimicrob. Agents Chemother. 61:e1573-17. doi: 10.1128/AAC.01573-17

Leid, J. G., Shirtliff, M. E., Costerton, J. W., and Stoodley, A. P. (2002). Human leukocytes adhere to, penetrate, and respond to Staphylococcus aureus biofilms. Infect. Immun. 70, 6339-6345. doi: 10.1128/iai.70.11.6339-6345. 2002
Lewis, K. (2010). Persister cells. Annu. Rev. Microbiol. 64, 357-372. doi: 10.1146/ annurev.micro.112408.134306

Li, H., Zhang, C., Liu, P., Liu, W., Gao, Y., and Sun, S. (2015). In vitro interactions between fluconazole and minocycline against mixed cultures of Candida albicans and Staphylococcus aureus. J. Microbiol. Immunol. Infect. 48, 655-661. doi: 10.1016/j.jmii.2014.03.010

Lin, J. N., Lai, C. H., Chen, Y. H., Chang, L. L., Lu, P. L., Tsai, S. S., et al. (2010). Characteristics and outcomes of polymicrobial bloodstream infections in the emergency department: a matched case-control study. Acad. Emerg. Med. 17, 1072-1079. doi: 10.1111/j.1553-2712.2010.00871.x

Maisonneuve, E., Castro-Camargo, M., and Gerdes, K. (2013). (p)ppGpp controls bacterial persistence by stochastic induction of toxin-antitoxin activity. Cell 154, 1140-1150. doi: 10.1016/j.cell.2013.07.048

McKenzie, F. E. (2006). Case mortality in polymicrobial bloodstream infections. J. Clin. Epidemiol. 59, 760-761. doi: 10.1016/j.jclinepi.2005.12.009

Monack, D. M., Mueller, A., and Falkow, S. (2004). Persistent bacterial infections: the interface of the pathogen and the host immune system. Nat. Rev. Microbiol. 2, 747-765. doi: 10.1038/nrmicro955

Odds, F. C., Brown, A. J., and Gow, N. A. (2004). Candida albicans genome sequence: a platform for genomics in the absence of genetics. Genome Biol. 5:230. doi: $10.1186 / \mathrm{gb}-2004-5-7-230$

Pereira, P. M., Filipe, S. R., Tomasz, A., and Pinho, M. G. (2007). Fluorescence imaging microscopy shows decreased access of vancomycin to cell wall synthetic sites in vancomycin-resistant Staphylococcus aureus. Antimicrob. Agents Chemother. 51, 3627-3633. doi: 10.1128/aac.00431-07

Perez, A. C., Pang, B., King, L. B., Tan, L., Murrah, K. A., Reimche, J. L., et al. (2014). Residence of Streptococcus pneumoniae and Moraxella catarrhalis within polymicrobial biofilm promotes antibiotic resistance and bacterial persistence in vivo. Pathog. Dis. 70, 280-288. doi: 10.1111/2049-632X.12129

Perlroth, J., Choi, B., and Spellberg, B. (2007). Nosocomial fungal infections: epidemiology, diagnosis, and treatment. Med. Mycol. 45, 321-346. doi: 10.1080/ 13693780701218689

Richardson, A. R., Somerville, G. A., and Sonenshein, A. L. (2015). Regulating the intersection of metabolism and pathogenesis in gram-positive bacteria. Microbiol. Spectr. 3. doi: 10.1128/microbiolspec.MBP-0004-2014

Royo-Cebrecos, C., Gudiol, C., Ardanuy, C., Pomares, H., Calvo, M., and Carratala, J. (2017). A fresh look at polymicrobial bloodstream infection in cancer patients. PLoS One 12:e0185768. doi: 10.1371/journal.pone.0185768

Shan, Y., Brown Gandt, A., Rowe, S. E., Deisinger, J. P., Conlon, B. P., and Lewis, K. (2017). ATP-dependent persister formation in Escherichia coli. mBio 8:e2267-16. doi: 10.1128/mBio.02267-16

Singh, R., Ray, P., Das, A., and Sharma, M. (2010). Penetration of antibiotics through Staphylococcus aureus and Staphylococcus epidermidis biofilms. J. Antimicrob. Chemother. 65, 1955-1958. doi: 10.1093/jac/dkq257

Spahich, N. A., Vitko, N. P., Thurlow, L. R., Temple, B., and Richardson, A. R. (2016). Staphylococcus aureus lactate- and malate-quinone oxidoreductases contribute to nitric oxide resistance and virulence. Mol. Microbiol. 100, 759773. doi: $10.1111 / \mathrm{mmi} .13347$

Thurlow, L. R., Joshi, G. S., and Richardson, A. R. (2018). Peroxisome proliferatoractivated receptor gamma is essential for the resolution of Staphylococcus aureus skin infections. Cell Host Microbe 24, 261.e4-270.e4. doi: 10.1016/j.chom.2018. 07.001

Tong, S. Y., Davis, J. S., Eichenberger, E., Holland, T. L., and Fowler, V. G. Jr. (2015). Staphylococcus aureus infections: epidemiology, pathophysiology, clinical manifestations, and management. Clin. Microbiol. Rev. 28, 603-661. doi: 10.1128/CMR.00134-14

Vitko, N. P., Spahich, N. A., and Richardson, A. R. (2015). Glycolytic dependency of high-level nitric oxide resistance and virulence in Staphylococcus aureus. mBio 6:e00045-15. doi: 10.1128/mBio.00045-15

Vuong, C., Voyich, J. M., Fischer, E. R., Braughton, K. R., Whitney, A. R., DeLeo, F. R., et al. (2004). Polysaccharide intercellular adhesin (PIA) protects Staphylococcus epidermidis against major components of the human innate immune system. Cell Microbiol. 6, 269-275. doi: 10.1046/j.1462-5822.2004. 00367.x

Wang, Y., Bojer, M. S., George, S. E., Wang, Z., Jensen, P. R., Wolz, C., et al. (2018). Inactivation of TCA cycle enhances Staphylococcus aureus persister cell formation in stationary phase. Sci. Rep. 8:10849. doi: 10.1038/s41598-01829123-0 
Wolcott, R. D., Rhoads, D. D., Bennett, M. E., Wolcott, B. M., Gogokhia, L., Costerton, J. W., et al. (2010). Chronic wounds and the medical biofilm paradigm. J. Wound Care 19, 45-46.

Zalis, E. A., Nuxoll, A. S., Manuse, S., Clair, G., Radlinski, L. C., Conlon, B. P., et al. (2019). Stochastic variation in expression of the tricarboxylic acid cycle produces persister cells. mBio 10:e1930-19. doi: 10.1128/mBio.019 30-19

Zarb, P., Coignard, B., Griskeviciene, J., Muller, A., Vankerckhoven, V., Weist, K., et al. (2012). The European centre for disease prevention and control (ECDC) pilot point prevalence survey of healthcare-associated infections and antimicrobial use. Euro Surveill. 17:20316.
Conflict of Interest: The authors declare that the research was conducted in the absence of any commercial or financial relationships that could be construed as a potential conflict of interest.

Copyright (c) 2019 Nabb, Song, Kluthe, Daubert, Luedtke and Nuxoll. This is an open-access article distributed under the terms of the Creative Commons Attribution License (CC BY). The use, distribution or reproduction in other forums is permitted, provided the original author(s) and the copyright owner(s) are credited and that the original publication in this journal is cited, in accordance with accepted academic practice. No use, distribution or reproduction is permitted which does not comply with these terms. 